Received: 25 January 2017

Accepted: 26 June 2017

Published online: 02 August 2017

\section{Metformin Inhibits Cyst Formation in a Zebrafish Model of Polycystin-2 Deficiency}

\author{
Ming-Yang Chang ${ }^{1}$, Tsu-Lin Ma ${ }^{1}$, Cheng-Chieh Hung ${ }^{1}$, Ya-Chung Tiann ${ }^{1}$, Yung-Chang Chen ${ }^{1}$, \\ Chih-WeiYang ${ }^{1} \&$ Yi-Chuan Cheng ${ }^{2}$
}

Autosomal dominant polycystic kidney disease (ADPKD) is a common kidney disease caused by mutations in PKD1 or PKD2. Metformin reduces cyst growth in mouse models of PKD1. However, metformin has not been studied in animal models of $P K D 2$, and the cellular mechanism underlying its effectiveness is not entirely clear. This study investigated the effects of metformin on cyst formation in a zebrafish model of polycystin-2 deficiency resulting from morpholino knockdown of $p k d 2$. We added metformin ( 2.5 to $20 \mathrm{mM}$ ) to the embryo media between 4 and 48 hours post fertilisation and observed pronephric cyst formation by using the wt1b promoter-driven GFP signal in $T g(w t 1 b: G F P) p k d 2$ morphants. Metformin inhibited pronephric cyst formation by $42-61 \%$ compared with the untreated controls. Metformin also reduced the number of proliferating cells in the pronephric ducts, the degree of dorsal body curvature, and the infiltration of leukocytes surrounding the pronephros. Moreover, metformin treatment increased the phosphorylation of adenosine monophosphate-activated protein kinase (AMPK) and enhanced autophagy in the pronephros. Our data suggest that metformin reduces cyst formation through activation of the AMPK pathway and modulation of defective cellular events such as proliferation and autophagy. These results also imply that metformin could have therapeutic potential for ADPKD treatment.

Autosomal dominant polycystic kidney disease (ADPKD) is a common genetic kidney disease resulting from mutations of $P K D 1$ or $P K D 2^{1}$. Affected patients may gradually lose their renal function because of progressive cyst formation in the kidneys; ADPKD accounts for $5 \%$ to $10 \%$ of cases of end-stage kidney disease (ESKD) ${ }^{2}$. Extrarenal presentations, such as liver cysts, cerebral aneurysms, and colon diverticulosis, can also cause serious complications. Patients with PKD2 mutations have a milder disease course than do those with PKD1 mutations. The ages at which patients with PKD1 and PKD2 mutations develop ESKD differ by almost 20 years ${ }^{3}$.

Polycystin-1 (encoded by PKD1) is a 4302-amino-acid membrane glycoprotein that is responsible for cell-cell or cell-matrix interactions critical for maintaining the renal tubular morphology ${ }^{4}$. Polycystin-2 (encoded by PKD2) is a 968-amino-acid cation ion channel involved in the regulation of intracellular calcium homeostasis, ${ }^{5,}$. Polycystin-1 and polycystin-2 can act synergistically or independently at the cell membrane, endoplasmic reticulum, and cilia, regulating cell proliferation, fluid secretion, extracellular matrix mechanics, and ciliary functions ${ }^{7}$.

The mechanism of cyst formation in ADPKD remains incompletely understood. Previous studies have shown that cyclic adenosine monophosphate (cAMP) and mammalian target of rapamycin (mTOR) are the major pathways involved in cell proliferation and fluid secretion in $\mathrm{ADPKD}^{8}$. Recent studies have suggested that enhanced aerobic glycolysis and defective autophagy in ADPKD cells may contribute to its hyperproliferative phenotype ${ }^{9}$. Furthermore, experimental evidence suggests that inflammation and macrophage infiltration have an additional role in promoting cystogenesis ${ }^{10}$. These novel discoveries of disease mechanisms have led to the investigation of many targeted therapies ${ }^{11-13}$. However, no treatment for ADPKD approved by the US Federal Drug Administration is currently available. A possible strategy to accelerate the discovery of new treatment is identifying new uses of existing drugs and repurposing them accordingly.

Metformin, an approved biguanide derivative, has been used for treating type 2 diabetes mellitus for decades ${ }^{14,15}$. Previous studies have shown that metformin inhibits gluconeogenesis through the activation of liver kinase B1

${ }^{1}$ Kidney Research Center and Department of Nephrology, Chang Gung Memorial Hospital, Chang Gung University College of Medicine, Taoyuan, Taiwan. ${ }^{2}$ Graduate Institute of Biomedical Sciences, College of Medicine, ChangGung University, Taoyuan, Taiwan. Correspondence and requests for materials should be addressed toY.-C.C. (email: yccheng@mail.cgu.edu.tw) 
A

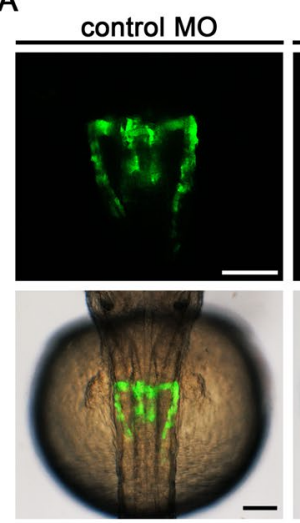

C

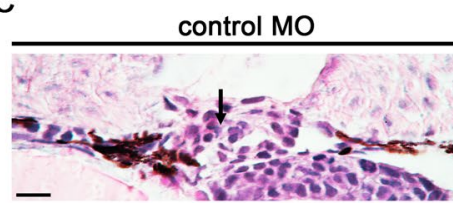

pkd2 MO

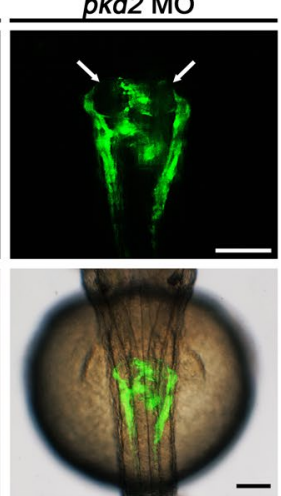

pkd2 MO+met $10 \mathrm{mM}$

pkd2 MO+met $10 \mathrm{mM}$

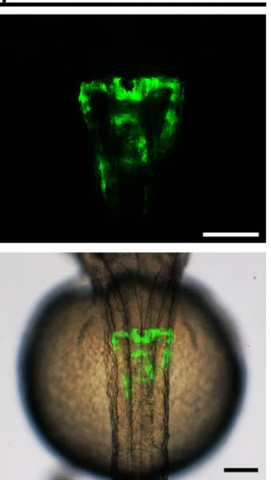

B

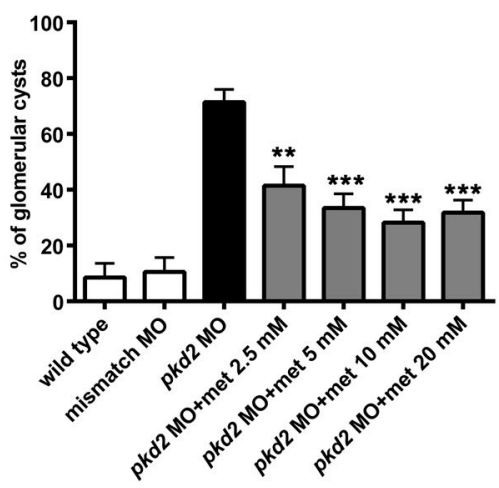

pkd2 MO

pkd2 MO+met $10 \mathrm{mM}$

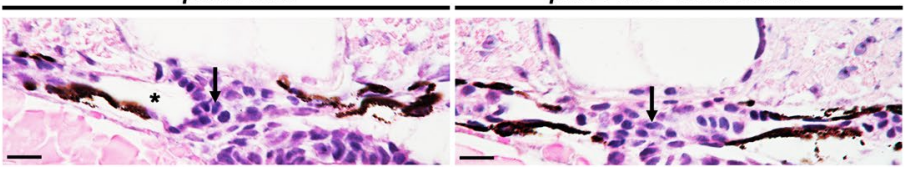

Figure 1. Metformin inhibits pronephric cyst formation in $p k d 2$ morphants. $T g(w t 1 b: G F P) p k d 2$ morphants were immersed in embryo media (E3) supplemented with 2.5, 5, 10, or $20 \mathrm{mM}$ metformin until 48 hpf. (A) Representative images of the pronephros in the control embryos, $p k d 2$ morphants, and metformin-treated $p k d 2$ morphants are shown. Note the pronephric cysts (arrows) in the $T g(w t 1 b: G F P) p k d 2$ morphant. Photographs from in vivo observation through fluorescence microscopy (dorsal view, anterior to the top) were recorded at $48 \mathrm{hpf}$. The lower panels show overlays of transmission (grey) and fluorescence (green) images of the upper panels. Scale bar, $100 \mu \mathrm{m}$. (B) Comparative frequencies of the cystic phenotype in the control embryos and $p k d 2$ morphants treated with different concentrations of metformin as indicated $(\mathrm{n}=45-99$ per group). Data represent five independent experiments. $* * P<0.01, * * * P<0.001$ compared with $p k d 2$ knockdown. (C)

Transverse histological sections of the embryos at $48 \mathrm{hpf}$, illustrating the pronephric cyst (indicated by the mark *) in a $p k d 2$ morphant and the rescue effect of metformin treatment. Arrows indicate the position of glomeruli. $\mathrm{H} \& \mathrm{E}$ stain, scale bar, $10 \mu \mathrm{m}$.

and adenosine monophosphate-activated protein kinase $(\mathrm{AMPK})^{16,17}$. In recent years, the therapeutic potential of metformin in cancer and polycystic ovary disease has drawn increasing attention ${ }^{18,19}$. For example, data from retrospective studies indicate that patients treated with metformin have a lower risk of prostate cancer and other urologic malignancies ${ }^{20}$, suggesting that metformin could have clinical applications in addition to glycemic control in type 2 diabetes mellitus ${ }^{21}$.

MacCarty et al. hypothesised that metformin can reduce cyst formation on the basis of its abilities to activate AMPK and suppress cystic fibrosis transmembrane conductance regulator (CFTR) and mTOR ${ }^{22}$. Accordingly, a previous study demonstrated that metformin inhibited cyst growth in Madin-Darby canine kidney (MDCK) cells and $P k d 1$ conditional knockout mice ${ }^{23}$. However, the exact in vivo mechanisms underlying the effect of metformin on cystogenesis are not entirely understood. Furthermore, recent studies have suggested that polycystin-1-deficent and polycystin-2-deficent cells might be different in the degree of AMPK inhibition ${ }^{24}$. Hence, whether metformin can inhibit cyst growth in the setting of polycystin-2 deficiency and the possible mechanisms of its action remain to be determined.

In this study, we investigated the effects of metformin on the initiation of pronephric cysts using a zebrafish $p k d 2$ model. We observed that metformin reduces pronephric cyst formation through the activation of AMPK and restores autophagy activity. These findings indicate that metformin could play a role in the treatment of early-stage ADPKD.

\section{Results}

Metformin prevents cyst initiation in pkd2 morphants. In this study, we used a zebrafish model of PKD2 for evaluating the effects of metformin on cyst initiation ${ }^{25-29}$. A translation-blocking morpholino oligos (MO) against $p k d 2$ was injected in a transgenic line, in which green fluorescent protein (GFP) expression was driven by the pronephros-specific $w t 1$ b promoter. We treated the $T g(w t 1 b: G F P) p k d 2$ morphants with different concentrations of metformin in the E3 buffer between 4 and 48 hours post fertilisation (hpf). As illustrated in Fig. 1A, metformin treatment resulted in a significant reduction (42\% to $61 \%$ ) of cyst formation in the $\operatorname{Tg}(w t 1 b: G F P) p k d 2$ morphants compared with untreated controls at $48 \mathrm{hpf}(P<0.01)$. The frequency of glomerular cyst formation decreased dose-dependently from $71.4 \% \pm 4.6 \%$ in the untreated $p k d 2$ morphants to $41.5 \% \pm 6.8 \%$ with $2.5 \mathrm{mM}$ metformin and $33.4 \% \pm 5.1 \%, 28.2 \% \pm 4.6 \%$, and $31.8 \% \pm 4.5 \%$ with $5 \mathrm{mM}, 10 \mathrm{mM}$, and $20 \mathrm{mM}$ metformin, respectively (Fig. 1B). In transverse histological sections, we confirmed the rescue effect of metformin treatment on the pronephric cysts in the $p k d 2$ morphants (Fig. 1C). These results indicate that metformin could inhibit the early stage of cyst formation in the zebrafish model of PKD2. 


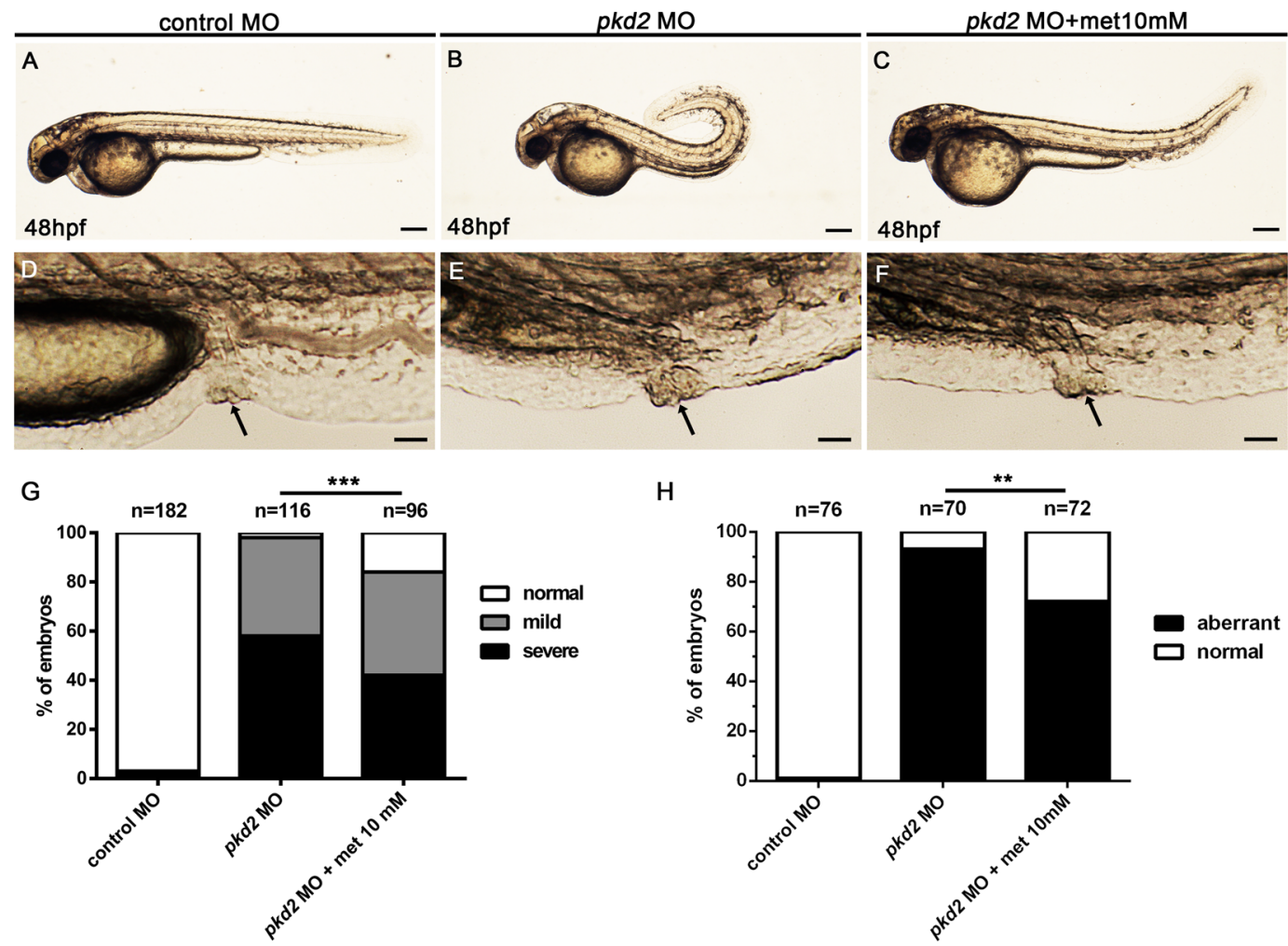

Figure 2. Metformin ameliorates dorsal axis curvature and cloaca malformation in $p k d 2$ morphants. Zebrafish embryos were treated with metformin $(10 \mathrm{mM})$ in supplemented E3 media. (A-C) Representative images showing the common body axis of a control embryo, the severe dorsal curvature of a $p k d 2$ morphant, and the mild dorsal curvature of a metformin-treated $p k d 2$ morphant. Scale bar, $200 \mu \mathrm{m}$. (D-F) Representative images showing the cloaca of a control embryo, the cloaca malformation of a $p k d 2$ morphant, and the normal cloaca of a metformin-treated $p k d 2$ morphant. Scale bar, $50 \mu \mathrm{m}$. (G) Comparative frequencies of the dorsal curvature phenotype in each group ( $\mathrm{n}=182,116$, and 96 per group). Data represent four independent experiments. $* * * P<0.001$ compared with $p k d 2$ morphants. (H) Comparative frequencies of the aberrant cloaca phenotype in each group $(\mathrm{n}=76,70$, and 72 per group). Data represent three independent experiments. $* * P<0.01$ compared with $p k d 2$ morphants.

Metformin reduces dorsal body curvature and cloaca malformation in pkd2 morphants. We examined the effect of metformin on the curvature phenotype of $p k d 2$ morphants (Fig. $2 \mathrm{~A}-\mathrm{C}$ ). Dorsal axis curvature results from an overproduction of type II collagen in the notochord sheath and has been used as a surrogate readout for cystogenesis in $p k d 2$ morphant ${ }^{26}$. As illustrated in Fig. $2 \mathrm{G}$, metformin partially reduced the frequencies of the overall curvature and severe dorsal curvature $\left(>90^{\circ}\right)$ phenotypes by $14 \%(114 / 116 \mathrm{vs.} 81 / 96, P<0.001)$ and $15 \%(67 / 116$ vs. $41 / 96, P<0.05)$, respectively, compared with untreated controls. These data indicate that metformin also ameliorated the defects in extracellular matrix formation involved in the pathogenesis of ADPKD. For comparison, we treated $p k d 1 a / b$ morphants with $10 \mathrm{mM}$ metformin and the curvature phenotype was also partially suppressed, similar to that observed in $p k d 2$ morphants (Supplementary Fig. S1). However, the small percentages of pronephric cysts in the $p k d 1 a / b$ morphants preclude us from further studies using this model ${ }^{30}$.

The cloaca malformation has been shown to correlate the formation of pronephric cysts in several zebrafish models of ciliopathies ${ }^{6,31-33}$. In agreement with these studies, we found that the frequency of aberrant cloaca increased in $p k d 2$ morphants and metformin significantly improved the phenotype by $21 \%(65 / 70$ vs. 52/72, $P<0.01)$ (Fig. 2D$\mathrm{F}, \mathrm{H})$. These data indicate that metformin consistently suppressed the different phenotypes of $p k d 2$ morphants. However, whether the improvement in cloacal morphology can lead to increased urine flow in metformin-treated $p k d 2$ morphants requires further study of kidney function using rhodamine-dextran filtration assays ${ }^{33-36}$.

Metformin reduces tubular cell proliferation. We next sought to determine whether metformin affects cell proliferation in the pronephric kidney. We performed double immunostaining using antibodies against phosphohistone $\mathrm{H} 3(\mathrm{PH} 3)$ and the $\mathrm{Na} / \mathrm{K}$-ATPase $\alpha-1$ subunit $(\alpha 6 \mathrm{~F})$ to mark proliferating cells in the pronephric ducts (Fig. 3A). Significantly fewer proliferating cells were observed in the pronephric ducts of the $p k d 2$ morphants treated with metformin than in those of the untreated $p k d 2$ morphants (Fig. $3 \mathrm{~B}$ ). These data suggest that metformin reduced pronephric cyst formation in the $p k d 2$ morphants through inhibition of epithelial cell proliferation. 

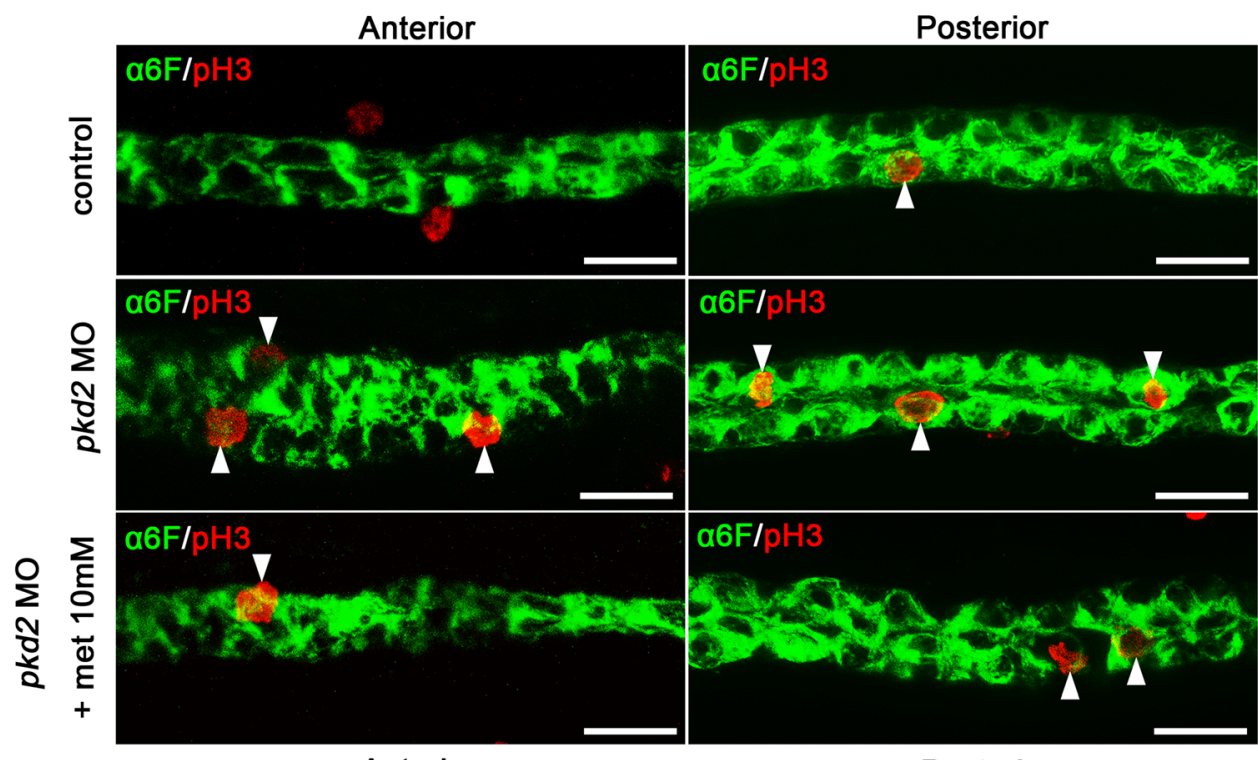

Anterior
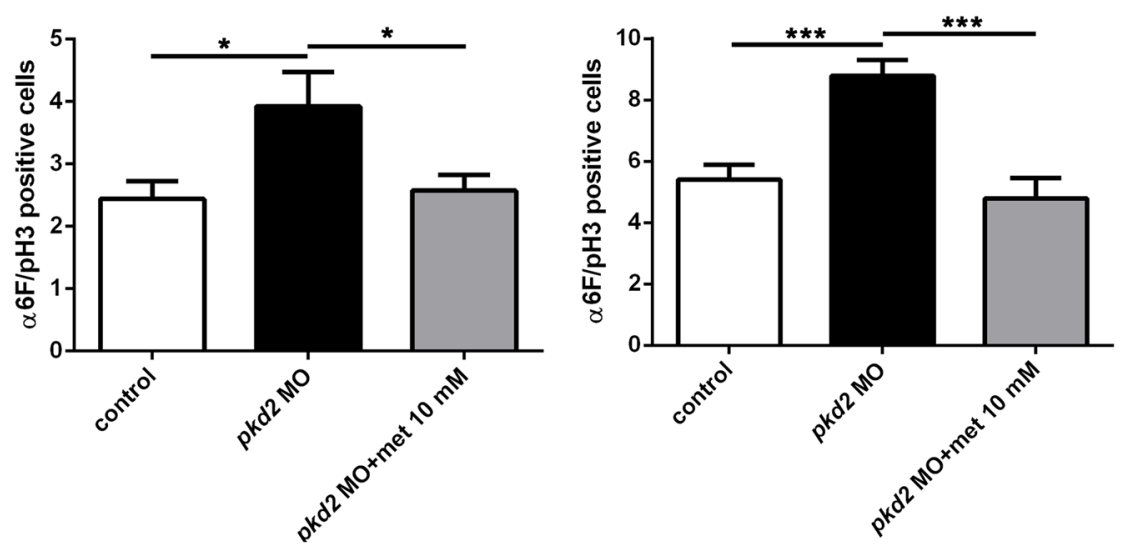

Figure 3. Metformin suppresses pronephric epithelial proliferation in $p k d 2$ morphants. (A) Representative confocal immunofluorescence images showing proliferative cells (arrows) in $p k d 2$ morphants with and without metformin $(10 \mathrm{mM})$ treatment. Embryos were stained using anti-PH3 (red) to mark proliferating cells and anti- $\alpha 6 \mathrm{~F}$ (green) to label the pronephric ducts at $48 \mathrm{hpf}$. (B) Counts of anti-PH3- and anti- $\alpha 6 \mathrm{~F}$-positive cells in the anterior and posterior pronephric ducts $(\mathrm{n}=14-17$ per group). $* P<0.05$, $* * * P<0.001$. Data represent two independent experiments. Scale bar, $20 \mu \mathrm{m}$.

Metformin reduces leukocyte accumulation. Previous studies have revealed that inflammation contributes to cell proliferation and cyst formation in $\mathrm{ADPKD}^{10}$. To explore the mechanisms by which metformin inhibits cyst growth, we analysed the effect of metformin on leukocyte infiltration in the pkd 2 morphants. Whole-mount in situ hybridisation revealed significantly increased l-plastin and mpx expression in the trunk area surrounding the pronephric ducts in the $p k d 2$ morphants compared with mismatched MO controls (Fig. 4A). The pan-leukocyte marker l-plastin is an actin-binding protein preferentially expressed in monocytes/macrophages and $m p x$ is a neutrophil marker ${ }^{37}$. Metformin treatment caused a significant reduction of leukocyte infiltration in the pronephric area in the $p k d 2$ morphants (Fig. $4 \mathrm{~B}$ and C). These data indicate that metformin could have an additional anti-inflammatory role that contributes to its therapeutic effects on polycystic kidney disease.

Activation of AMPK by metformin. A previous study revealed that $P k d 1^{-1-}$ mouse embryonic fibroblasts exhibit a lower level of AMPK phosphorylation than do wild-type cells ${ }^{9}$. Metformin has been shown to stimulate AMPK in cell and mouse models of ADPKD ${ }^{23,24}$. Therefore, we evaluated the effect of metformin on the activation of the AMPK signalling pathway. Western blot analysis using an antibody against p-AMPK $\alpha$ at Thr172 indicated that the $\mathrm{p}$-AMPK/AMPK ratio was significantly higher in the metformin-treated $p k d 2$ morphants than in the untreated controls (Fig. 5A and B, Supplementary Fig. S2). These data are consistent with the hypothesis that AMPK activation inhibits polycystic kidney disease. However, we did not observe a lower baseline expression level of $\mathrm{p}$-AMPK in the $p k d 2$ morphants than in the wild-type controls; this effect could be partly due to the presence of residual maternal pAMPK in the zebrafish embryos ${ }^{38}$. 
A

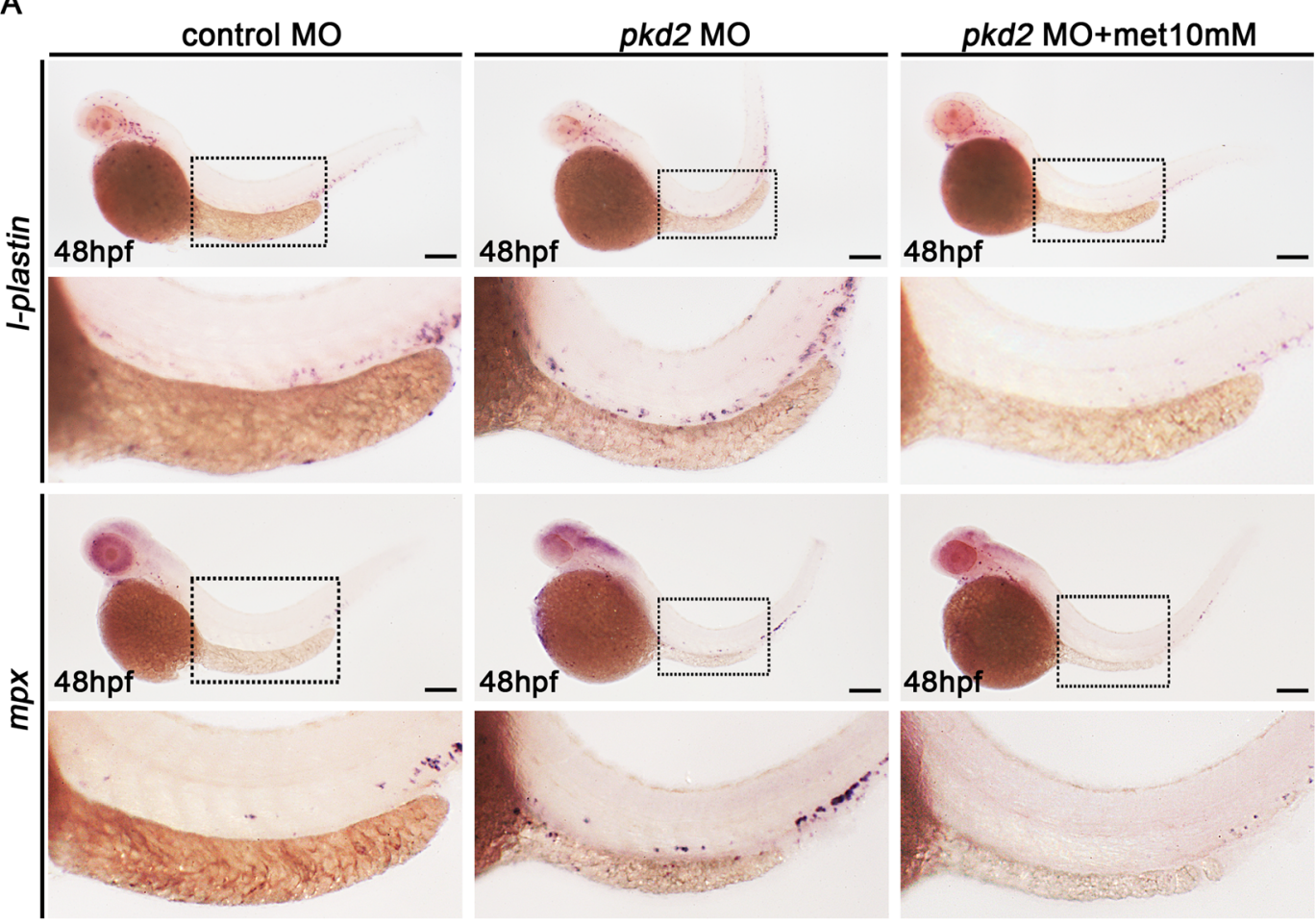

B

C
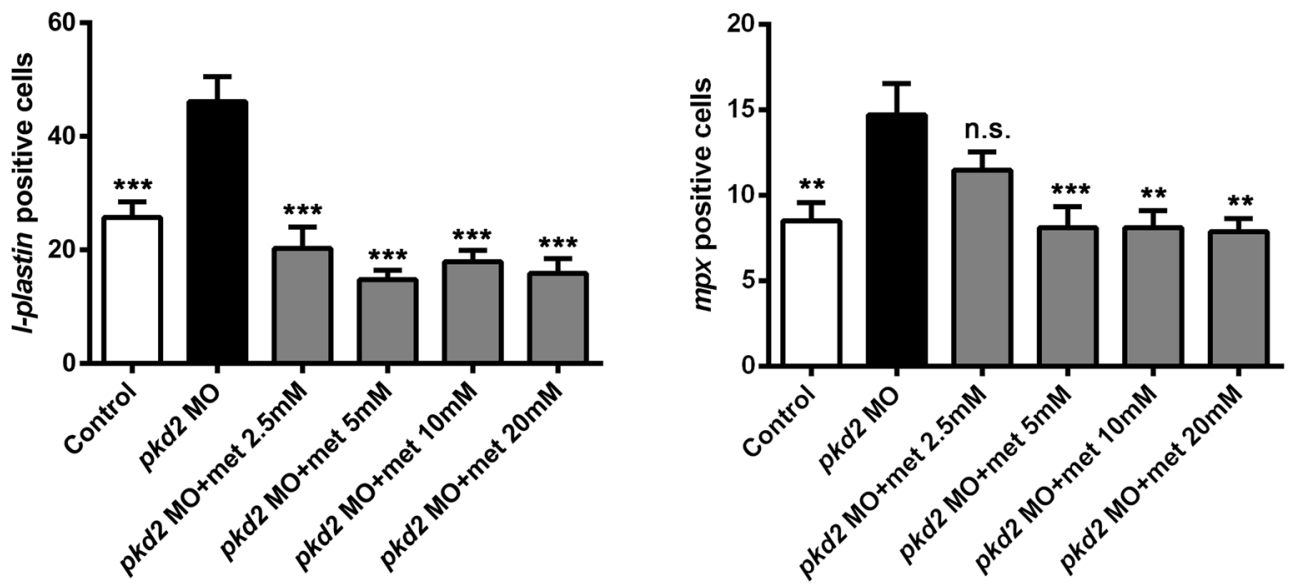

Figure 4. Metformin reduces leukocyte infiltration in $p k d 2$ morphants. (A) Representative images of $l$-plastinpositive and $m p x$-positive cells surrounding the pronephric ducts in a control MO-injected embryo, a $p k d 2$ morphant, and a $p k d 2$ morphant treated with metformin $(10 \mathrm{mM})$. Leukocytes were labelled using in situ hybridisation for l-plastin and $m p x$ at $48 \mathrm{hpf}$. The lower panel shows an enlarged view of the dashed box indicated in the upper image. Scale bar, $200 \mu \mathrm{m}$. (B) Counts of the l-plastin-positive cells (within the region of yolk extension as indicated by the dashed box $)$ in the controls and metformin-treated $p k d 2$ morphants $(2.5 \mathrm{mM}$ to $20 \mathrm{mM})$. $* * * P<0.001$ compared with $p k d 2$ morphants. Data represent two independent experiments, $\mathrm{n}=7-11$ per group. (C) Counts of the $m p x$-positive cells. $* * P<0.01, * * * P<0.001$ compared with pkd2 morphants. n.s. $=$ not significant. Data represent two independent experiments, $n=16-20$ per group.

Metformin cannot prevent cyst formation in tsc1a morphants. A study indicated that the AMPK-dependent activation of tuberous sclerosis complex (TSC) 1 and 2 proteins plays a major role in the inhibition of the mTOR signalling pathway during energetic demands ${ }^{39}$. Therefore, we tested whether metformin prevented cyst initiation in the absence of TSC proteins. Morpholino knockdown of tsc1a induced severe glomerular cysts in zebrafish embryos at $48 \mathrm{hpf}$, as reported previously (Fig. 6A) ${ }^{40}$. Metformin apparently did not affect cyst formation in the tscla morphants (Fig. 6B). This observation is consistent with an AMPK/TSC-dependent mechanism underlying the prevention of cyst formation by metformin. 

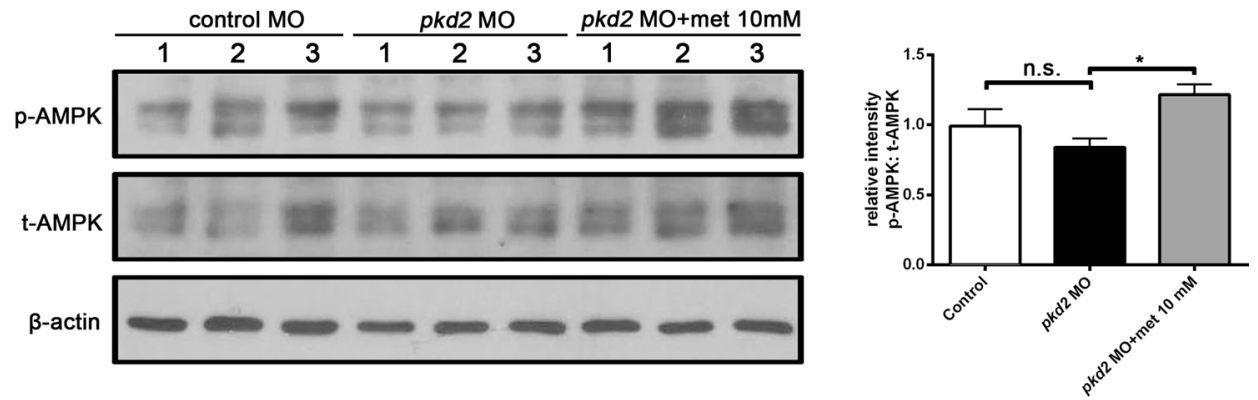

Figure 5. Metformin increases AMPK levels in $p k d 2$ morphants. (A) Representative Western blots for phospho-Thr ${ }^{172}$ AMPK, total AMPK, and $\beta$-actin in total embryo lysates from mismatch MO-injected controls and pkd2 morphants with and without metformin $(10 \mathrm{mM})$ treatment at $48 \mathrm{hpf}$. (B) Quantification of $\mathrm{p}$-AMPK relative to total AMPK through densitometric analysis of Western blots; $\mathrm{n}=9$ from five independent experiments. $* P<0.05$, n.s. $=$ not significant. Cropped blot images are shown, and all the blots were run under the same experimental conditions. Uncropped blots are shown in Supplementary Fig. S2.

Metformin enhances autophagy in the pronephros. Defective autophagy, an AMPK downstream cellular process that degrades cytoplasmic components for energy production, has been associated with the pathogenesis of ADPKD in recent studies ${ }^{41}$. Therefore, we examined whether metformin increased the autophagy activities in $p k d 2$ morphants. Autophagy activity was assessed by detecting the intracellular levels of microtubule-associated protein light chain 3 (LC3) through immunofluorescence and Western blot analysis ${ }^{42}$. As illustrated in Fig. 7A, the pkd2 morphants exhibited lower LC3 staining in the pronephric ducts than did the control embryos, and the deficiency was rectified by metformin treatment. Consistent with these findings, the Western blot analysis indicated that the conversion of cytoplasmic LC3-I into LC3-II was significantly lower in the $p k d 2$ morphants than in the control embryos, and metformin treatment restored the autophagy activities (Fig. 7B and Supplementary Fig. S2). Furthermore, we observed that simultaneous knockdown of the autophagy gene atg 5 significantly increased the cystic phenotype in the $p k d 2$ morphants (Fig. 7C and D). Although the magnitudes of these differences were moderate and further studies are needed to confirm our findings, these results suggest that the activation of AMPK and restoration of autophagy constitute a plausible mechanism for the inhibition of cyst growth by metformin ${ }^{43}$.

\section{Discussion}

The zebrafish has become an increasingly recognised model for identifying drug candidates for ADPKD treatment ${ }^{44-47}$. Chemical compound screening in zebrafish $p k d 2$ models indicated that histone deacetylase inhibitors inhibited cyst growth ${ }^{48}$. Candidate drugs that are effective in other PKD animal models, including rapamycin, roscovitine, and pasireotide, have been successfully validated using the zebrafish system ${ }^{34,49}$. In the current study, we demonstrated that metformin activated the phosphorylation of AMPK and prevented cyst formation in a zebrafish $p k d 2$ model. In particular, we demonstrated that metformin inhibited epithelial cell proliferation and restored normal autophagy activity in the pronephric kidney. Furthermore, we demonstrated that metformin did not prevent cyst formation in tscla morphants resulting from knocking down the AMPK downstream target TSC1.

Metformin was shown to suppress cyst growth and fluid secretion through the inhibition of mTOR and CFTR in a previous study using MDCK cell cysts, mouse embryonic kidney explant cultures, and $P k d 1$ mouse models ${ }^{23}$. In accordance with this study, we found that metformin inhibited early cystogenesis in zebrafish $p k d 2$ morphant embryos, indicating that metformin can inhibit cyst growth in both PKD1 and PKD2 animal models. These results support the hypothesis that metformin reduces cyst formation in the early stages of ADPKD. Interestingly, a previous study suggests that polycystin-2 deficient cell lines are less amenable to metformin and rapamycin than polycystin-1 deficient cell lines due to the less activation of mTOR pathway ${ }^{24}$. However, we observed a similar suppressive effect of metformin on the curvature phenotype in both $p k d 1 a / b$ and $p k d 2$ morphants. The discrepancy could be explained by the differences between in vivo and in vitro cystic models, or metformin could have pleiotropic effects on multiple signalling pathways related to the PKD2 deficiency. Further studies will be required to clarify our findings.

One possible mechanism by which metformin prevents cyst formation is through the activation of AMPK and inhibition of cell proliferation. The AMPK signalling pathway plays a crucial role in maintaining normal kidney structure during nephron morphogenesis by affecting cell proliferation and migration ${ }^{50,51}$. Activated AMPK restores the cell energy balance by shutting down the ATP-consuming synthesis pathways, and thus causes cells to switch from an anabolic to a catabolic state ${ }^{18}$. Metformin reduced cell proliferation in an AMPK-dependent manner in MDCK cysts grown in 3D collagen gels ${ }^{23}$. Similarly, intraperitoneal injection of metformin resulted in a reduction in the number of Ki67-positive epithelial cells in the cystic kidneys of $P k d 1^{f l o x /}-K s p$-Cre mice at postnatal day $7^{23}$. Furthermore, forced activation of AMPK by 2-deoxyglucose (2DG) restored normal extracellular signal-regulated kinase (ERK) activity, inhibited glycolysis, and reduced the cystic index and proliferation rate in 
A

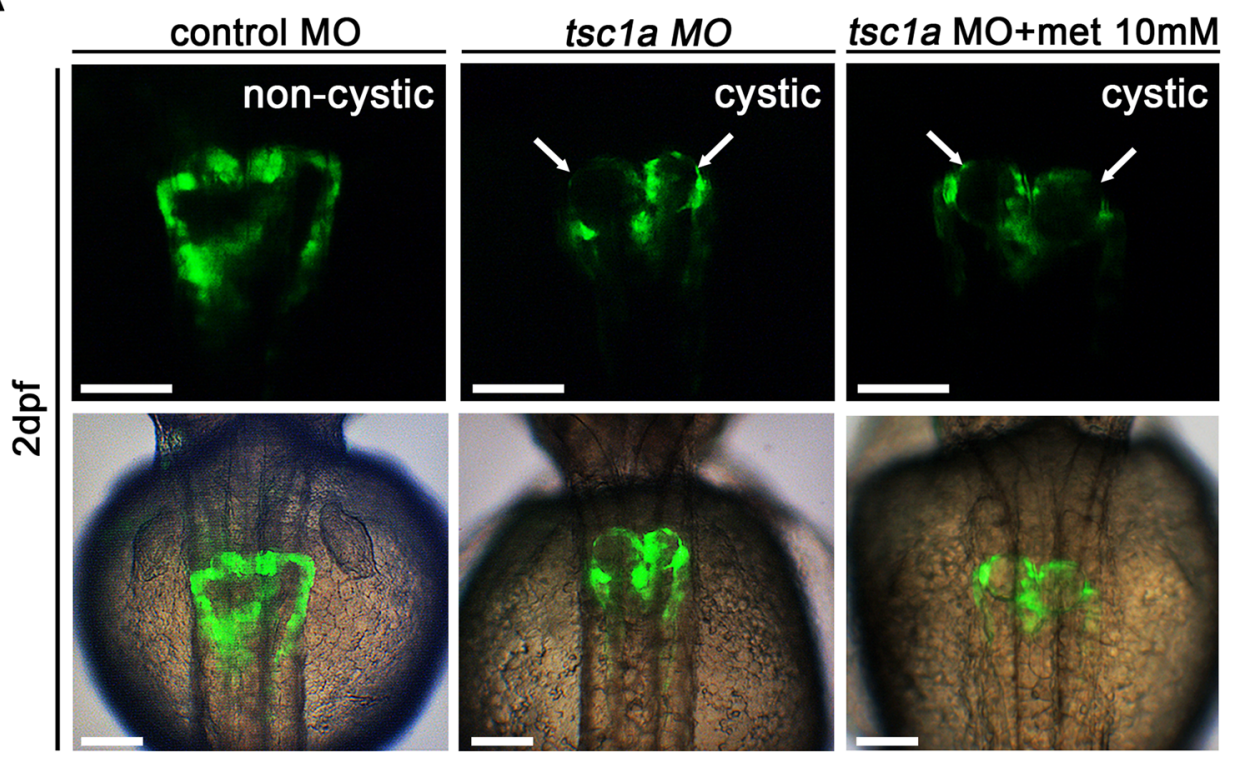

B

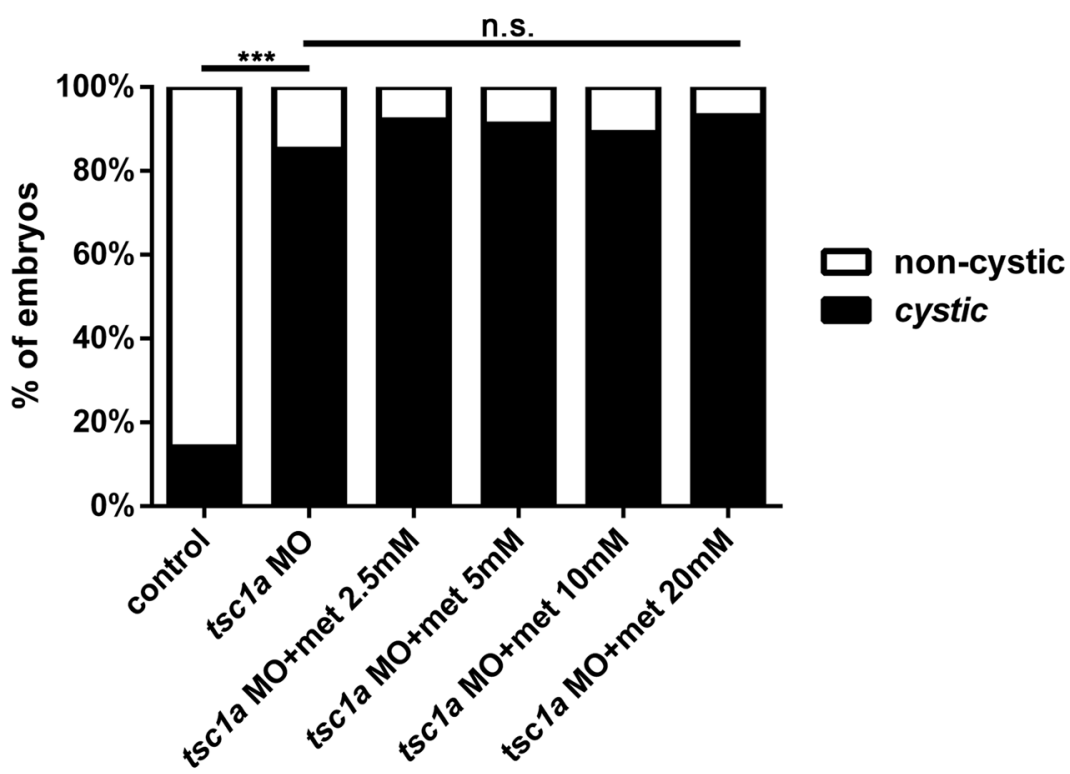

Figure 6. Metformin cannot rescue the cystic phenotype in $t s c 1 a$ morphants. $T g(w t 1 b: G F P) t s c 1 a$ morphants were immersed in embryo media (E3) containing 2.5 to $20 \mathrm{mM}$ metformin until $48 \mathrm{hpf}$. (A) Representative images indicating prominent cystic pronephros (arrows) in $\operatorname{Tg}(w t 1 b: G F P) t s c 1 a$ morphants. Metformin does not affect the formation and severity of pronephric cysts. The upper panels show fluorescence images (dorsal view, anterior to the top). The lower panels show overlays of fluorescence (green) and transmission (grey) images of the upper panels. Scale bar, $100 \mu \mathrm{m}$. (B) Comparison of the cystic phenotype in different treatment groups. Data represent two independent experiments $(\mathrm{n}=28-46$ per group $) * * * P<0.001$, n.s. $=$ not significant.

$P k d 1$ conditional knockout mice ${ }^{9}$. Our findings are consistent with these results and suggest that metformin may inhibit cystogenesis through an AMPK-dependent pathway.

Growing evidence substantiates the role of macrophages in promoting cyst growth in ADPKD. Macrophages were demonstrated to stimulate cell proliferation and cyst expansion in coculture experiments within a collagen matrix ${ }^{52}$. The recruitment and retention of renal macrophages contributes to the increased proliferation during cyst growth in $\mathrm{ADPKD}^{10}$. A previous study showed that metformin treatment reduced inflammatory cytokine production in peripheral blood mononuclear cells obtained from healthy volunteers ${ }^{53}$. Metformin also suppressed inflammation through activation of AMPK and phosphatase and tensin homolog (PTEN) in vascular smooth muscle cells ${ }^{54}$. In accordance with these findings, we observed that metformin reduced the recruitment of macrophages in $p k d 2$ morphants, which could be an alternative mechanism through which metformin reduces cell proliferation and cyst formation. 
A

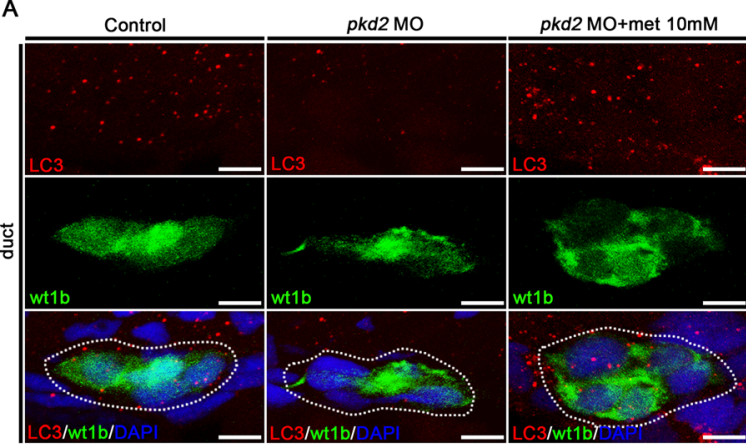

C

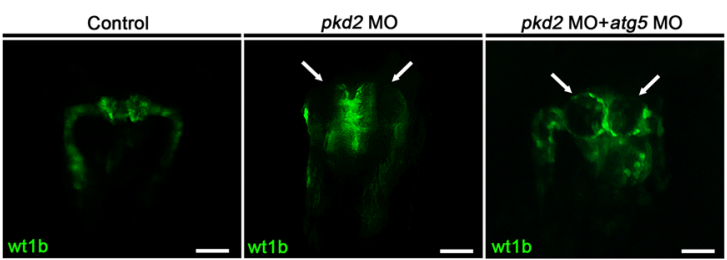

B
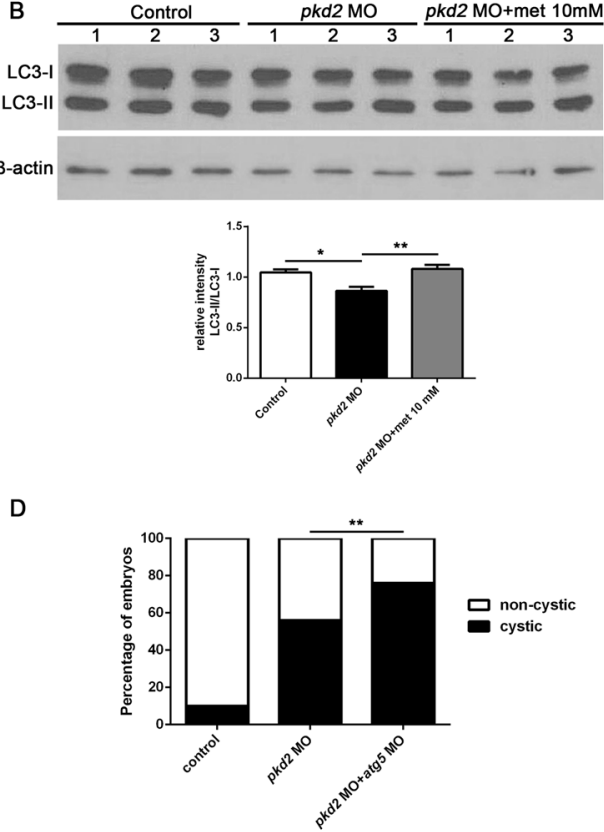

Figure 7. Metformin enhances autophagy in $T g(w t 1 b: G F P) p k d 2$ morphants. (A) Representative confocal images of pronephric ducts (green, wt $1 b: G F P$ ) in control MO-injected embryos, $p k d 2$ morphants, and $p k d 2$ morphants treated with metformin $(10 \mathrm{mM})$ at $48 \mathrm{hpf}(\mathrm{n}=4$ per group). Transverse cryosections were stained for LC3 (red), and the nuclei were counterstained with DAPI (blue). Note the reduced expression of LC3 (orange dots, red and green merged) in the $p k d 2$ morphant and the rescue effect of metformin treatment. The dotted circle indicates the pronephric duct. Scale bar, $10 \mu \mathrm{m}$. (B) Quantification of the expression of LC3-II relative to LC3-I through Western blot analysis of total embryo lysates. $B$-actin was used as a loading control; $\mathrm{n}=8$ from four independent experiments. $* P<0.05$ compared with control; $* * P<0.01$ compared with untreated $p k d 2$ morphants. The uncropped blots are shown in Supplementary Fig. S2. (C) A combined knockdown of atg 5 and $p k d 2$ increased the frequency of cystic phenotype in the $\operatorname{Tg}(w t 1 b: G F P) p k d 2$ morphants. Representative images of the pronephros in the control embryos, $p k d 2$ morphants, and atg5/pkd2 double morphants are shown. Arrows indicate the pronephric cysts. (D) Comparison of the cystic phenotype in different groups. Data represent four independent experiments $(\mathrm{n}=81,86$, and 87 per group). $* * P<0.01$.

Another significant finding in this study is that metformin corrects the defective autophagy observed in $p k d 2$ morphants. Autophagy is a highly regulated cellular process that degrades and recycles intracellular proteins and organelles in lysosomes during metabolic stress or nutritional deprivation ${ }^{55}$. Our finding that $p k d 2$ morphants had defective autophagy is consistent with a previous study showing that $P k d 1^{-1-}$ mouse embryonic fibroblasts had insufficient autophagy activity upon glucose deprivation ${ }^{9}$. Furthermore, our results suggested that metformin might reduce cyst formation through the enhancement of autophagy in ADPKD ${ }^{56}$. Autophagy suppression could reduce the senescence of cyst-lining cells and lead to increased proliferation, apoptosis, and cyst growth ${ }^{57}$. Autophagy has also been shown to regulate the formation and function of primary cilia ${ }^{58}$. The detailed mechanisms that link autophagy and cyst growth require further study ${ }^{59}$.

The similarities in the cystic phenotype of $p k d 2$ and $t s c 1 a$ morphants prompted us to investigate whether metformin could also inhibit cyst formation in this previously described model of tuberous sclerosis ${ }^{40}$. However, our results demonstrated that metformin could not rescue the cystic phenotype of the tsc la morphants. This observation is consistent with an AMPK-dependent effect of metformin because TSC1 is a known downstream target of AMPK. Long-term treatment with metformin also failed to suppress renal tumours in a $T s c 1^{+/-}$mouse model ${ }^{60}$. We were unable to further determine the relative contribution of AMPK activation in the inhibition of cystogenesis because of a lack of a proper morpholino or specific antagonist to reduce AMPK activity without interfering with the development of zebrafish embryos ${ }^{38}$. Therefore, we cannot exclude the possibility that metformin inhibits cystogenesis through an AMPK-independent effect ${ }^{18,61}$.

Our data contribute to a growing body of evidence that metabolic abnormalities are crucial in the pathogenesis of $\mathrm{ADPKD}^{50,62}$. Inhibition of aerobic glycolysis by using 2DG suppressed cell proliferation, leukocyte infiltration, and cyst formation in mouse models of $\mathrm{PKD1}^{9}$. Mild to moderate food restriction was demonstrated to activate AMPK and reduce cyst area, renal fibrosis, and inflammation ${ }^{63}$. These studies support a new therapeutic strategy for ADPKD, which involves reprogramming of cellular metabolism ${ }^{64}$.

Although our results support the hypothesis that metformin is a potential treatment for ADPKD, metformin could cause lactic acidosis in patients with advanced renal failure ${ }^{18}$. Therefore, the inhibitory effect of metformin on cyst growth in animal models requires confirmation in future clinical studies. A randomised, double-blind 
clinical trial evaluating metformin in the treatment of patients with ADPKD was started in 2016 and is expected to be completed in the next few years (ClinicalTrials.gov Identifier: NCT02656017).

In conclusion, metformin reduces cyst formation in $p k d 2$-deficient zebrafish embryos. The data suggest that metformin may prevent cyst formation through activation of the AMPK pathway and modulation of defective cellular events such as proliferation and autophagy. The findings also indicate the therapeutic potential of metformin in treatment of ADPKD at the early stages. Further studies are required to assess whether metformin can improve clinical outcomes in patients with ADPKD.

\section{Methods}

Zebrafish maintenance. Animal experiments were approved by the Chang Gung University Institutional Animal Care and Use Committee. The investigation conformed to the National Institutes of Health Guide for the Care and Use of Laboratory Animals. Zebrafish and embryos were maintained according to standard procedures $^{65}$. The embryos were staged according to hours post fertilisation. We used the $\operatorname{Tg}(w t 1 b$ :GFP) line (kindly provided by Prof. Christoph Englert, Fritz Lipmann Institute, Jena, Germany) for in vivo observation of pronephric cysts ${ }^{25-27,66}$.

Morpholino injection. Zebrafish embryos at the one- or two-cell stage were microinjected with $0.125 \mathrm{mM}$ antisense MO. MOs were obtained from Gene Tools (Philomath, OR) and had the following sequences: $p k d 2$ ATG-MO (5'-AGGACGAACGCGACTGGAGCTCATC- $\left.3^{\prime}\right)^{29}, p k d 2$ 5-mismatch MO (5'-AGCACCAACCCGACTGCACCTCATC-3'), tsc 1 a ATG-MO (5'-CCATAGTTGTGCAGGACAGTGGGCA $\left.-3^{\prime}\right)^{40}$, tscla 5-mismatch MO (5'-CCATACTTCTGCAGCACACTGGCCA-3'), pkd1a exon 8 splice-MO MO: (5'-GATCTGAGGACTCACTGTGTGATTT- $\left.3^{\prime}\right)^{30}$, pkd $1 b$ exon 45 splice-MO MO: $\left(5^{\prime}-\mathrm{AC}\right.$ ATGATATTTGTACCTCTTTGGTT-3 $\left.{ }^{\prime}\right)^{30}$, and $\operatorname{atg} 5$ ATG-MO (5'-CACATCCTTGTCATCTGCCATTATC-3 $\left.{ }^{\prime}\right)^{67}$.

Drug treatment for zebrafish. $\quad P k d 2$ morphant embryos $(\mathrm{n}=20-30)$ were incubated in 6-cm Petri dishes with $8 \mathrm{~mL}$ of the $\mathrm{E} 3$ medium $\left(5 \mathrm{mM} \mathrm{NaCl}, 0.17 \mathrm{mM} \mathrm{KCl}, 0.4 \mathrm{mM} \mathrm{CaCl}_{2}\right.$, and $\left.0.16 \mathrm{mM} \mathrm{MgSO}_{4}\right)$ in an incubator at $28.5^{\circ} \mathrm{C}$. Metformin (Sigma) was added to the $\mathrm{E} 3$ medium at final concentrations of 5 to $20 \mathrm{mM}$ at $4 \mathrm{hpf}$, a time point before the development of body curvature and pronephric cysts according to previous studies ${ }^{30,48}$. At 48 hpf, the living embryos were anaesthetised with tricaine $(0.2 \mathrm{mg} / \mathrm{mL}$ ) and oriented in $3 \%$ methylcellulose (Sigma). The presence of pronephric cysts was determined using fluorescence microscopy ${ }^{25-27}$. The dorsal body curvature phenotype of $p k d 2$ morphants was categorised as normal, mild (tail angle less than $90^{\circ}$ ), or severe (tail curvature angle greater than $\left.90^{\circ}\right)^{48}$.

Immunofluorescence. Embryos were fixed overnight in $4 \%$ paraformaldehyde at $4{ }^{\circ} \mathrm{C}$. Immunostaining was performed in whole-mount embryos as described previously ${ }^{68}$. A rabbit anti-PH3 antibody (1:200, Millipore) was used to label proliferating cells, and a mouse anti- $\alpha 6 \mathrm{~F}$ antibody (1:200, Developmental Studies Hybridoma Bank) was used to label pronephric epithelial cells. LC3, an autophagosome marker, was labelled using a rabbit anti-LC3B antibody (1:200, Novus Biologicals). Secondary antibodies used were the Alexa Fluor 594 goat antirabbit IgG and Alexa Fluor 488 goat antimouse IgG (1:500, Molecular Probes). Proliferating cells in the pronephric ducts were counted in anti-PH3 and anti- $\alpha 6 \mathrm{~F}$ stained whole-mount embryos using fluorescence microscopy. For confocal imaging, embryos were flat-mounted and the alpha $6 \mathrm{~F}$ and $\mathrm{PH} 3$ signals were recorded in $\mathrm{z}$-series stacks using a Zeiss LSM 510 confocal microscope. For analysis of LC3 staining, the immunostained embryos were embedded in the OTC medium and cryosectioned through the pronephros. The sections were mounted in Vectashield (Vector Laboratories) with DAPI and imaged using confocal microscopy.

Histology. The embryos were fixed in $4 \%$ paraformaldehyde overnight at $4{ }^{\circ} \mathrm{C}$ and embedded in glycolmethacrylate (JB-4; Polyscience). Serial sections $(4 \mu \mathrm{m})$ were cut and stained with Hematoxylin and Eosin (H \& E).

Whole-mount in situ hybridisation. The embryos were fixed overnight in $4 \%$ paraformaldehyde at $4{ }^{\circ} \mathrm{C}$. Whole-mount in situ hybridisation was performed according to published protocols ${ }^{65}$. Antisense digoxigenin-labelled RNA probes were synthesised from linearised plasmid templates containing $l$-plastin and $m p x$ cDNAs.

Western blot analysis. Proteins were extracted from whole embryos for Western blot analysis using standard protocols ${ }^{38,69}$. Primary antibodies, namely anti-phospho-AMPK $\alpha$ (Thr172) (1:1000, Cell Signalling), anti-AMPK $\alpha$ (1: 1000, Cell Signalling), anti-LC3B (1:10000, Novus Biologicals), and anti- $\beta$-actin (AC-15) (1: 10000, Abcam) antibodies, were used overnight at $4{ }^{\circ} \mathrm{C}$. Horseradish peroxidase-conjugated secondary antibodies were used for $1 \mathrm{~h}$ at room temperature. The signals were detected through enhanced chemiluminescence.

Statistical analysis. Values are expressed as the mean \pm SEM. Comparisons between groups were performed using the Student's $t$ test or ANOVA followed by Dunnett's multiple comparison test. Values that were not normally distributed were analysed using the Kruskal-Wallis test followed by Dunnett's multiple comparison test. Categorical variables were analysed using Fisher's exact test or the chi-square test. $P$ values less than 0.05 were considered statistically significant. All analyses were performed using GraphPad Prism 5.0 (GraphPad, La Jolla, CA, USA). 


\section{References}

1. Heyer, C. M. et al. Predicted Mutation Strength of Nontruncating PKD1 Mutations Aids Genotype-Phenotype Correlations in Autosomal Dominant Polycystic Kidney Disease. J Am Soc Nephrol 27, 2872-2884, doi:10.1681/ASN.2015050583 (2016).

2. Ong, A. C., Devuyst, O., Knebelmann, B. \& Walz, G. Autosomal dominant polycystic kidney disease: the changing face of clinical management. Lancet 385, 1993-2002, doi:10.1016/S0140-6736(15)60907-2 (2015).

3. Cornec-Le Gall, E. et al. Type of PKD1 mutation influences renal outcome in ADPKD. J Am Soc Nephrol 24, 1006-1013, doi:10.1681/ ASN.2012070650 (2013).

4. Talbot, J. J. et al. The cleaved cytoplasmic tail of polycystin-1 regulates Src-dependent STAT3 activation. J Am Soc Nephrol 25, 1737-1748, doi:10.1681/ASN.2013091026 (2014).

5. Geng, L. et al. Polycystin-2 traffics to cilia independently of polycystin-1 by using an N-terminal RVxP motif. J Cell Sci 119 , 1383-1395, doi:10.1242/jcs.02818 (2006).

6. Obara, T. et al. Polycystin-2 immunolocalization and function in zebrafish. J Am Soc Nephrol 17, 2706-2718, doi:10.1681/ ASN.2006040412 (2006).

7. Ong, A. C. \& Harris, P. C. A polycystin-centric view of cyst formation and disease: the polycystins revisited. Kidney Int 88, 699-710, doi:10.1038/ki.2015.207 (2015).

8. Harris, P. C. \& Torres, V. E. Genetic mechanisms and signaling pathways in autosomal dominant polycystic kidney disease. J Clin Invest 124, 2315-2324, doi:10.1172/JCI72272 (2014).

9. Rowe, I. et al. Defective glucose metabolism in polycystic kidney disease identifies a new therapeutic strategy. Nat Med 19, 488-493, doi:10.1038/nm.3092 (2013).

10. Chen, L. et al. Macrophage migration inhibitory factor promotes cyst growth in polycystic kidney disease. J Clin Invest $\mathbf{1 2 5}$, 2399-2412, doi:10.1172/JCI80467 (2015).

11. Torres, V. E., Gansevoort, R. T. \& Czerwiec, F. S. Tolvaptan in autosomal dominant polycystic kidney disease. N Engl J Med 368, 1259, doi:10.1056/NEJMc1300762 (2013).

12. Walz, G. et al. Everolimus in patients with autosomal dominant polycystic kidney disease. N Engl J Med 363, 830-840, doi:10.1056/ NEJMoa1003491 (2010).

13. Serra, A. L. et al. Sirolimus and kidney growth in autosomal dominant polycystic kidney disease. N Engl J Med 363, 820-829, doi:10.1056/NEJMoa0907419 (2010).

14. Zhou, G. et al. Role of AMP-activated protein kinase in mechanism of metformin action. JClin Invest 108, 1167-1174, doi:10.1172/ JCI13505 (2001).

15. Wander, S. A., Hennessy, B. T. \& Slingerland, J. M. Next-generation mTOR inhibitors in clinical oncology: how pathway complexity informs therapeutic strategy. J Clin Invest 121, 1231-1241, doi:10.1172/JCI44145 (2011).

16. Shaw, R. J. et al. The kinase LKB1 mediates glucose homeostasis in liver and therapeutic effects of metformin. Science $\mathbf{3 1 0}$, 1642-1646, doi:10.1126/science.1120781 (2005).

17. Viollet, B. et al. Targeting the AMPK pathway for the treatment of Type 2 diabetes. Front Biosci (Landmark Ed) 14, 3380-3400 (2009).

18. Viollet, B. et al. Cellular and molecular mechanisms of metformin: an overview. Clin Sci (Lond) 122, 253-270, doi:10.1042/ CS20110386 (2012).

19. Kim, H. G. et al. Metformin inhibits P-glycoprotein expression via the NF-kappaB pathway and CRE transcriptional activity through AMPK activation. Br J Pharmacol 162, 1096-1108, doi:10.1111/j.1476-5381.2010.01101.x (2011).

20. Sayyid, R. K. \& Fleshner, N. E. Potential role for metformin in urologic oncology. Investig Clin Urol 57, 157-164, doi:10.4111/ icu.2016.57.3.157 (2016).

21. Pollak, M. Overcoming Drug Development Bottlenecks With Repurposing: Repurposing biguanides to target energy metabolism for cancer treatment. Nat Med 20, 591-593, doi:10.1038/nm.3596 (2014).

22. McCarty, M. F., Barroso-Aranda, J. \& Contreras, F. Activation of AMP-activated kinase as a strategy for managing autosomal dominant polycystic kidney disease. Med Hypotheses 73, 1008-1010, doi:10.1016/j.mehy.2009.05.043 (2009).

23. Takiar, V. et al. Activating AMP-activated protein kinase (AMPK) slows renal cystogenesis. Proc Natl Acad Sci USA 108, 2462-2467, doi:10.1073/pnas.1011498108 (2011).

24. Mekahli, D. et al. Polycystin-1 but not polycystin-2 deficiency causes upregulation of the mTOR pathway and can be synergistically targeted with rapamycin and metformin. Pflugers Arch 466, 1591-1604, doi:10.1007/s00424-013-1394-x (2014).

25. Chang, M. Y. et al. Inhibition of the P2X7 receptor reduces cystogenesis in PKD. J Am Soc Nephrol 22, 1696-1706, doi:10.1681/ ASN.2010070728 (2011).

26. Le Corre, S., Eyre, D. \& Drummond, I. A. Modulation of the secretory pathway rescues zebrafish polycystic kidney disease pathology. J Am Soc Nephrol 25, 1749-1759, doi:10.1681/ASN.2013101060 (2014).

27. Arif Pavel, M. et al. Function and regulation of TRPP2 ion channel revealed by a gain-of-function mutant. Proc Natl Acad Sci USA 113, E2363-2372, doi:10.1073/pnas.1517066113 (2016).

28. Schottenfeld, J., Sullivan-Brown, J. \& Burdine, R. D. Zebrafish curly up encodes a Pkd2 ortholog that restricts left-side-specific expression of southpaw. Development 134, 1605-1615, doi:10.1242/dev.02827 (2007).

29. Sun, Z. et al. A genetic screen in zebrafish identifies cilia genes as a principal cause of cystic kidney. Development 131, 4085-4093, doi:10.1242/dev.01240 (2004).

30. Mangos, S. et al. The ADPKD genes pkd1a/b and pkd2 regulate extracellular matrix formation. Dis Model Mech 3, 354-365, doi:10.1242/dmm.003194 (2010).

31. Bubenshchikova, E. et al. Wtip and Vangl2 are required for mitotic spindle orientation and cloaca morphogenesis. Biol Open 1, 588-596, doi:10.1242/bio.20121016 (2012).

32. Slanchev, K., Putz, M., Schmitt, A., Kramer-Zucker, A. \& Walz, G. Nephrocystin-4 is required for pronephric duct-dependent cloaca formation in zebrafish. Hum Mol Genet 20, 3119-3128, doi:10.1093/hmg/ddr214 (2011).

33. Rothschild, S. C., Francescatto, L., Drummond, I. A. \& Tombes, R. M. CaMK-II is a PKD2 target that promotes pronephric kidney development and stabilizes cilia. Development 138, 3387-3397, doi:10.1242/dev.066340 (2011).

34. Tobin, J. L. \& Beales, P. L. Restoration of renal function in zebrafish models of ciliopathies. Pediatr Nephrol 23, 2095-2099, doi:10.1007/s00467-008-0898-7 (2008).

35. Xu, W. et al. The Joubert Syndrome Protein Inpp5e Controls Ciliogenesis by Regulating Phosphoinositides at the Apical Membrane. J Am Soc Nephrol 28, 118-129, doi:10.1681/ASN.2015080906 (2017).

36. Kramer-Zucker, A. G. et al. Cilia-driven fluid flow in the zebrafish pronephros, brain and Kupffer's vesicle is required for normal organogenesis. Development 132, 1907-1921, doi:10.1242/dev.01772 (2005).

37. de Jong, J. L. \& Zon, L. I. Use of the zebrafish system to study primitive and definitive hematopoiesis. Annu Rev Genet 39, 481-501, doi:10.1146/annurev.genet.39.073003.095931 (2005).

38. Mendelsohn, B. A., Kassebaum, B. L. \& Gitlin, J. D. The zebrafish embryo as a dynamic model of anoxia tolerance. Dev Dyn 237, 1780-1788, doi:10.1002/dvdy.21581 (2008).

39. Choo, A. Y. et al. Glucose addiction of TSC null cells is caused by failed mTORC1-dependent balancing of metabolic demand with supply. Mol Cell 38, 487-499, doi:10.1016/j.molcel.2010.05.007 (2010).

40. DiBella, L. M., Park, A. \& Sun, Z. Zebrafish Tscl reveals functional interactions between the cilium and the TOR pathway. Hum Mol Genet 18, 595-606, doi:10.1093/hmg/ddn384 (2009). 
41. De Rechter, S. et al. Autophagy in renal diseases. Pediatr Nephrol 31, 737-752, doi:10.1007/s00467-015-3134-2 (2016).

42. Lenoir, O., Tharaux, P. L. \& Huber, T. B. Autophagy in kidney disease and aging: lessons from rodent models. Kidney Int 90, 950-964, doi:10.1016/j.kint.2016.04.014 (2016).

43. Zhu, P., Sieben, C. J., Xu, X., Harris, P. C. \& Lin, X. Autophagy activators suppress cystogenesis in an autosomal dominant polycystic kidney disease model. Hum Mol Genet 26, 158-172, doi:10.1093/hmg/ddw376 (2017).

44. Drummond, I. A. Kidney development and disease in the zebrafish. J Am Soc Nephrol 16, 299-304, doi:10.1681/ASN.2004090754 (2005).

45. Sussman, C. R. et al. Phosphodiesterase 1A modulates cystogenesis in zebrafish. J Am Soc Nephrol 25, 2222-2230, doi:10.1681/ ASN.2013040421 (2014).

46. Zhou, X. et al. SIRT2 regulates ciliogenesis and contributes to abnormal centrosome amplification caused by loss of polycystin-1. Hum Mol Genet 23, 1644-1655, doi:10.1093/hmg/ddt556 (2014).

47. Wu, M. et al. Resveratrol delays polycystic kidney disease progression through attenuation of nuclear factor kappaB-induced inflammation. Nephrol Dial Transplant 31, 1826-1834, doi:10.1093/ndt/gfw058 (2016).

48. Cao, Y. et al. Chemical modifier screen identifies HDAC inhibitors as suppressors of PKD models. Proc Natl Acad Sci USA 106, 21819-21824, doi:10.1073/pnas.0911987106 (2009).

49. Tietz Bogert, P. S. et al. The zebrafish as a model to study polycystic liver disease. Zebrafish 10, 211-217, doi:10.1089/zeb.2012.0825 (2013).

50. Menezes, L. F. et al. Network analysis of a Pkd1-mouse model of autosomal dominant polycystic kidney disease identifies HNF4alpha as a disease modifier. PLoS Genet 8, e1003053, doi:10.1371/journal.pgen.1003053 (2012).

51. Vasilyev, A., Liu, Y., Hellman, N., Pathak, N. \& Drummond, I. A. Mechanical stretch and PI3K signaling link cell migration and proliferation to coordinate epithelial tubule morphogenesis in the zebrafish pronephros. PLoS One 7, e39992, doi:10.1371/journal. pone.0039992 (2012).

52. Swenson-Fields, K. I. et al. Macrophages promote polycystic kidney disease progression. Kidney Int 83, 855-864, doi:10.1038/ ki.2012.446 (2013)

53. Buldak, L. et al. Metformin affects macrophages' phenotype and improves the activity of glutathione peroxidase, superoxide dismutase, catalase and decreases malondialdehyde concentration in a partially AMPK-independent manner in LPS-stimulated human monocytes/macrophages. Pharmacol Rep 66, 418-429, doi:10.1016/j.pharep.2013.11.008 (2014).

54. Kim, S. A. \& Choi, H. C. Metformin inhibits inflammatory response via AMPK-PTEN pathway in vascular smooth muscle cells. Biochem Biophys Res Commun 425, 866-872, doi:10.1016/j.bbrc.2012.07.165 (2012)

55. Klionsky, D. J. \& Emr, S. D. Cell biology - Autophagy as a regulated pathway of cellular degradation. Science 290, 1717-1721, doi:10.1126/science.290.5497.1717 (2000).

56. Hardie, D. G. AMPK and autophagy get connected. EMBO J 30, 634-635, doi:10.1038/emboj.2011.12 (2011)

57. Ravichandran, K. \& Edelstein, C. L. Polycystic kidney disease: a case of suppressed autophagy? Semin Nephrol 34, 27-33, doi:10.1016/j.semnephrol.2013.11.005 (2014).

58. Pampliega, O. \& Cuervo, A. M. Autophagy and primary cilia: dual interplay. Curr Opin Cell Biol 39, 1-7, doi:10.1016/j. ceb.2016.01.008 (2016).

59. Chen, N., Eritja, N., Lock, R. \& Debnath, J. Autophagy restricts proliferation driven by oncogenic phosphatidylinositol 3-kinase in three-dimensional culture. Oncogene 32, 2543-2554, doi:10.1038/onc.2012.277 (2013).

60. Yang, J., Kalogerou, M., Gallacher, J., Sampson, J. R. \& Shen, M. H. Renal tumours in a Tsc1 +/- mouse model show epigenetic suppression of organic cation transporters Slc22a1, Slc22a2 and Slc22a3, and do not respond to metformin. Eur J Cancer 49, 1479-1490, doi:10.1016/j.ejca.2012.10.027 (2013).

61. Cameron, A. R. et al. Anti-Inflammatory Effects of Metformin Irrespective of Diabetes Status. Circ Res 119, 652-665, doi:10.1161/ CIRCRESAHA.116.308445 (2016).

62. Menezes, L. F., Lin, C. C., Zhou, F. \& Germino, G. G. Fatty Acid Oxidation is Impaired in An Orthologous Mouse Model of Autosomal Dominant Polycystic Kidney Disease. EBioMedicine 5, 183-192, doi:10.1016/j.ebiom.2016.01.027 (2016).

63. Warner, G. et al. Food Restriction Ameliorates the Development of Polycystic Kidney Disease. J Am Soc Nephrol 27, 1437-1447, doi:10.1681/ASN.2015020132 (2016)

64. Rowe, I. \& Boletta, A. Defective metabolism in polycystic kidney disease: potential for therapy and open questions. Nephrol Dial Transplant 29, 1480-1486, doi:10.1093/ndt/gft521 (2014).

65. Westerfield, M. The zebrafish book. A guide for the laboratory use of zebrafish (Danio rerio), 4th ed. University of Oregon Press, Eugene, OR (2000).

66. Perner, B., Englert, C. \& Bollig, F. The Wilms tumor genes wt1a and wt $1 \mathrm{~b}$ control different steps during formation of the zebrafish pronephros. Dev Biol 309, 87-96, doi:10.1016/j.ydbio.2007.06.022 (2007).

67. Lee, E. et al. Autophagy is essential for cardiac morphogenesis during vertebrate development. Autophagy 10, 572-587, doi:10.4161/ auto.27649 (2014).

68. Krens, S. F. et al. Distinct functions for ERK1 and ERK2 in cell migration processes during zebrafish gastrulation. Dev Biol 319, 370-383, doi:10.1016/j.sydbio.2008.04.032 (2008).

69. Santoriello, C. et al. Expression of H-RASV12 in a zebrafish model of Costello syndrome causes cellular senescence in adult proliferating cells. Dis Model Mech 2, 56-67, doi:10.1242/dmm.001016 (2009).

\section{Acknowledgements}

The authors thank Yi-Hui Huang for technical assistance. This work was supported by grants from Chang Gung Memorial Hospital (CMRPG3D0651, CMRPG3E2001) and the Ministry of Science and Technology of Taiwan (102-2314-B-182-045, 104-2314-B-182A-114).

\section{Author Contributions}

M.-Y.C. and Y.-C. Cheng conceived and designed the experiments; T.-L.M. performed the experiments; C.-C.H., Y.-C.T., Y.-C. Chen, and C.-W.Y. analysed the data; and M.-Y.C. and Y.-C. Cheng wrote the paper. All authors reviewed the manuscript.

Additional Information

Supplementary information accompanies this paper at doi:10.1038/s41598-017-07300-X

Competing Interests: The authors declare that they have no competing interests.

Publisher's note: Springer Nature remains neutral with regard to jurisdictional claims in published maps and institutional affiliations. 
(i) Open Access This article is licensed under a Creative Commons Attribution 4.0 International License, which permits use, sharing, adaptation, distribution and reproduction in any medium or format, as long as you give appropriate credit to the original author(s) and the source, provide a link to the Creative Commons license, and indicate if changes were made. The images or other third party material in this article are included in the article's Creative Commons license, unless indicated otherwise in a credit line to the material. If material is not included in the article's Creative Commons license and your intended use is not permitted by statutory regulation or exceeds the permitted use, you will need to obtain permission directly from the copyright holder. To view a copy of this license, visit http://creativecommons.org/licenses/by/4.0/.

(C) The Author(s) 2017 